\title{
Improved Delivery of CRISPR/Cas9 System Using Magnetic Nanoparticles into Porcine Fibroblast
}

\author{
Magdalena Hryhorowicz ${ }^{1}$ Bartosz Grześkowiak ${ }^{2}$. Natalia Mazurkiewicz ${ }^{1}$ Paweł Śledziński ${ }^{1}$. Daniel Lipiński ${ }^{1}$. \\ Ryszard Słomski ${ }^{1,3}$
}

Published online: 17 December 2018

(c) The Author(s) 2018

\begin{abstract}
Genetically modified pigs play an important role in agriculture and biomedical research; hence, new efficient methods are needed to obtain genetically engineered cells and animals. The clustered regularly interspaced short palindromic repeats (CRISPR)/Cas (CRISPR-associated) system represents an effective genome editing tool. It consists of two key molecules: single guide RNA (sgRNA) and the Cas9 endonuclease that can be introduced into the cells as one plasmid. Typical delivery methods for CRISPR/Cas9 components are limited by low transfection efficiency or toxic effects on cells. Here, we describe the use of magnetic nanoparticles and gradient magnetic field to improve delivery of CRISPR/Cas 9 constructs into porcine fetal fibroblasts. Polyethylenimine-coated nanoparticles with magnetic iron oxide core were used to form magnetic plasmid DNA lipoplexes. CRISPR/Cas9 construct was prepared to induce site-specific cutting at the porcine H11 locus. Quantitative assessment of genomic cleavage by sequence trace decomposition demonstrated that the magnetofection efficiency was more than 3.5 times higher compared to the classic lipofection method. The Tracking of Indels by Decomposition web tool precisely determined the spectrum of indels that occurred. Simultaneously, no additional cytotoxicity associated with the utilization of magnetic nanoparticles was observed. Our results indicate that magnetofection enables effective delivery of the CRISPR/Cas9 construct into porcine fetal fibroblasts with low cell toxicity.
\end{abstract}

Keywords CRISPR/Cas9 delivery · Magnetic nanoparticles $\cdot$ Magnetofection $\cdot$ Porcine fetal fibroblasts · Transfection

\section{Introduction}

Since exploiting the clustered regularly interspaced short palindromic repeats (CRISPR)/CRISPR-associated protein 9 (Cas9) systems for DNA editing in 2012 [1], CRISPR/Cas9 technology has become one of the major genomic engineering tools and has been tested in a wide range of cell lines and organisms. Due to its high efficiency, versatility and simplicity, the CRISPR/Cas9 system has been recognized as the most promising strategy for many biomedical applications,

Magdalena Hryhorowicz

b_a-b_y@wp.pl

1 Department of Biochemistry and Biotechnology, Poznan University of Life Sciences, Dojazd 11, 60-632 Poznan, Poland

2 The NanoBioMedical Centre, Adam Mickiewicz University, Umultowska 85, 61-614 Poznan, Poland

3 Institute of Human Genetics, Polish Academy of Sciences, Strzeszyńska 32, 60-101 Poznan, Poland such as gene therapy, xenotransplantation and using animals as models for studying human diseases [2]. One of the conditions for successful cell modification is the use of an efficient delivery method for single-guide RNA (sgRNA) and the enzyme Cas9. Generally, components of the CRISPR/Cas9 system can be introduced into cells as DNA (in the form of a plasmid) or mRNA. The plasmid DNA format is a popular and attractive delivery approach due to its stability, simplicity and low cost of conducting experiments. However, compared to the mRNA form, the use of plasmid decreases the genome editing efficiency [3]. One of the most popular techniques for CIRPSR/Cas9-mediated genome editing is the utilization of viral vectors. Despite their high transduction efficiency, viruses have some disadvantages, such as the risk of cytotoxicity and immunogenicity, expensive largescale production and limited insertion size, as well as the risk of integrating viral sequences into the target genome [4]. Therefore, non-viral delivery systems for CRISPR/ Cas9 are a promising alternative. Typical non-viral transfection approaches for in vitro conditions include physical 
(electroporation or nucleofection) and chemical delivery methods (lipofection or polyfection). Electroporation and nucleofection disrupt cellular barriers by a strong electric field. The increased permeability of the cell membrane by electrical impulses enables high transfection efficiency in various cell types, but may have a negative impact on cell viability $[5,6]$. Chemical delivery techniques utilize cationic lipids or cationic polymers as enhancers to induce membrane poration or endocytosis, as well as to protect DNA/RNA from degradation. Although lipofection and polyfection have the advantages of ease of use, low cost and greater safety compared with viral delivery methods, they are also limited by unsatisfactory transfection efficiency $[4,7]$. Currently, non-viral delivery routes for the CRISPR/Cas 9 components via the use of nanoparticles (NPs) have the potential to overcome these limitations. The advantages of nanoparticles are their nanoscale size, high surface area, stability and biocompatibility. In recent years, various non-viral nanoparticles, such as cell-penetrating peptide, cationic arginine gold nanoparticles and graphene oxide-polyethylene glycol-polyethylenimine (PEI) have been successfully used for delivering CRISPR/Cas9 components into different cell types [8-10].

In this study, we decided to test the potential of magnetic nanoparticles (MNPs) to improve the delivery of CRISPR/ Cas9 plasmid into porcine fetal fibroblasts (PFFs). This method, called magnetofection, is based on the delivery of nucleic acid vectors associated with MNPs under the influence of an external magnetic field into the cells. Magnetofection uses a magnetic force to sediment and concentrate the vectors onto the surface of the cells to be transfected $[11,12]$. We also showed that using MNPs to deliver a gene construct into porcine fibroblast cells greatly improves transfection efficacy [13]. Genetically modified pigs are widely used in biomedicine as animal models of various human diseases as well as for xenotransplantation purpose, due to their similarity to human physiology and anatomy [14, 15]. The applications of the CRISPR/Cas9 system for generating site-specific gene knockout/knockin in a pig model may significantly accelerate the advance of biomedical science. Therefore, new, efficient and safe delivery methods for CRISPR/Cas9 components are needed. In this study, we used magnetofection technology to deliver CRISPR/Cas9 constructs containing a sequence encoding a Cas 9 protein and sgRNA for generating site-specific cutting at the H11 locus into PFFs. This porcine "safe harbor" genomic locus, named $\mathrm{pH} 11$, is transcriptionally active and enables genetic modifications without disrupting internal gene function [16]. To form magnetic plasmid DNA lipoplexes, iron oxide nanoparticles with magnetic core and polyethylenimine coating (PEI-Mag2 NPs) were used. The transfected cells were subjected to further genomic cleavage detection assays. To evaluate the efficiency of the magnetofection, PCR and sequencing analysis were performed. Moreover, to estimate the cytotoxicity of the PEI-Mag2 MNPs on PFFs, sensitive colorimetric assays were used.

\section{Materials and Methods}

\section{CRISPR/Cas9 Plasmid Preparation}

The sgRNA targeting the pH11 locus was designed using online CRISPR design tools-http://crispr.mit.edu/. Two complementary oligonucleotides carrying a sequence to the target site and BbsI-compatible overhangs were annealed and inserted into BbsI sites of a pSpCas9(BB)-2A-Puro (PX459) V2.0 vector using the protocol described by Ran et al. [17]. The overall strategy is shown in Fig. 1. The PX459 vector containing both sgRNA and the Cas9 sequences was a gift from Feng Zhang (Addgene plasmid \#62988). The correctness of cloned oligonucleotides was confirmed by means of sequencing using automated genetic analyzers (Applied Biosystems Prism). The prepared CRISPR/Cas9 construct was purified by the Endo Free Plasmid Purification Maxi Kit (Qiagen).

\section{Cell Culture}

PFFs isolated from conceptuses were cultured in Dulbecco's modified Eagle medium (DMEM, Sigma-Aldrich) supplemented with $10 \%$ fetal bovine serum (FBS, Sigma-Aldrich), $1 \%$ antibiotic/antimycotic solution (Sigma-Aldrich) and cultivated in culture flasks at $37{ }^{\circ} \mathrm{C}$ in a humidified atmosphere containing 5\% $\mathrm{CO}_{2}$. The medium was replaced every 3-4 days. Cell growth and morphology were assessed using a Zeiss Axiovert 200 inverted microscope. Cultures of $80 \%$ confluence were split 1:2 and seeded in a fresh medium into new culture flasks. The cell lines used in transfection did not exceed five passages.

\section{Magnetic Nanoparticles}

PEI-Mag2 core/shell-type nanoparticles with a magnetic iron oxide core and PEI coating were kindly donated by Dr. Olga Mykhaylyk (ethris GmbH). Briefly, PEI-Mag2 MNPs were synthesized by precipitation of $\mathrm{Fe}(\mathrm{II}) / \mathrm{Fe}$ (III) hydroxide from an aqueous salt solution, followed by transformation into magnetite in an oxygen-free atmosphere, with immediate spontaneous adsorption of the $25 \mathrm{kDa}$ branched polyethylenimine (PEI-25Br) (Sigma-Aldrich) combined with the fluorinated surfactant ZONYL FSA (lithium 3-[2(perfluoroalkyl)ethylthio]propionate) (Sigma-Aldrich), as described elsewhere [18]. 


\section{(A)}

GATGGATTGGGATTCAGGTCTCTGCTGTTGTTACTTGAGATTGCTTTCTAGATTCTACTTGTGGAAA CAAAAAGCCTTTGCGAGAATTCTAAACTGGAGTATTTCTGTAATTGAGGAGTCTTGCTCAGCAAAT CCCACTTAGGGGACTAATGAAGTACCAGGAAGAGACAGACCATGCTCAATCCACAAAGCCAGGTT TTACTGAAATGTGACCTACTTTCTTATGTTCCTGGAAGTTTAGATCAGGGTGGGCAGCTCTGGGTTT TATAGGCTACACTGTTAACACTCAGGCTGTTTTCTACCGTTTAGTCAAAATATAGTCACCTTGCCTG CTTCACCTGTCCATCAGAGAATGGCCTCATTAATTGACTCTCTAGTATGAAGTCAAAGTAGCTTTGG TGGCCCTAAATGGACAAGTATCAAGAGACTGGGTGAATTGAGGAGCTT

(B)

\section{5'- CACCGTTCCTGGAAGTTTAGATCA -3’ \\ 5'- AAACTGATCTAAACTTCCAGGAAC -3'}

(C)

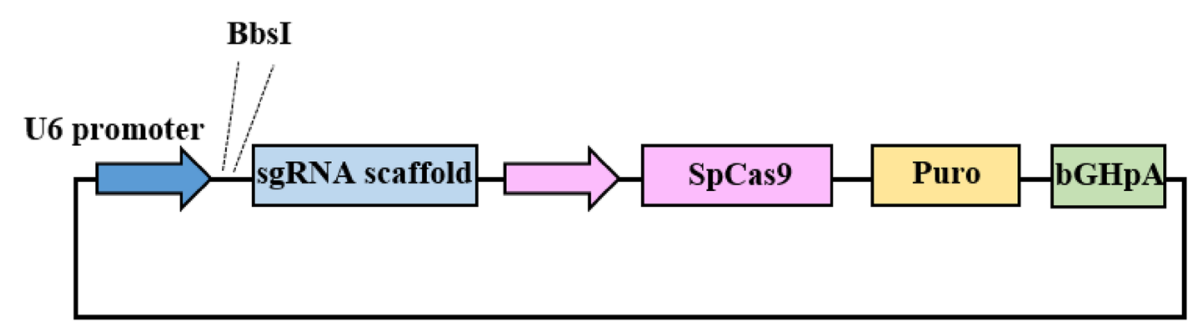

Fig. 1 Design and preparation of the CRISPR/Cas9 plasmid. a H11 locus and target site for sgRNA (target site in red and Protospacer Adjacent Motif in green font). b Oligonucleotides designed

\section{Preparation of the Transfection Complexes}

Magnetic complexes were prepared by mixing PEI-Mag2 MNPs with transfection reagent DreamFect Gold (DF-Gold, OZ Biosciences) and CRISPR/Cas9 plasmid in serum- and supplement-free DMEM at a transfection reagent-to-plasmid DNA ratio of 4:1 (v/w) and an iron-to-plasmid DNA ratio of 2:1 (w/w). In order to assemble the components, the mixture was incubated for $20 \mathrm{~min}$ at room temperature. Lipoplexes used as a reference were prepared using the same method with water instead of a magnetic nanoparticle suspension.

\section{Cell Transfection Using Magnetofection}

One day prior to transfection, PFFs were plated onto sixwell plates at a seeding density of $7.5 \times 10^{4}$ cell/well in $4 \mathrm{ml}$ of growth medium, providing a confluence of $50 \%$ on the day of transfection. One hundred microliters of the freshly prepared magnetic complexes was added to each well, resulting in a CRISPR/Cas 9 construct dose of 10 or 20 pg DNA/ cell. The plate was positioned on the Mega Magnetic Plate (OZ Biosciences) and incubated at $37^{\circ} \mathrm{C}$ for $20 \mathrm{~min}$. At this time, the magnetic complexes were sedimented onto the cell surface. Next, the Mega Magnetic Plate was removed and the cell culture plate was incubated for $24 \mathrm{~h}$. As a reference, for sgRNA targeting the $\mathrm{pH} 11$ locus with BbsI-compatible to vector overhangs (blue font). c Cas9 from S. pyogenes with 2A-Puro, and cloning backbone for sgRNA. (Color figure online)

lipofection of the PFFs was performed in parallel. The experiment was carried out in triplicate.

At $24 \mathrm{~h}$ post-transfection, the cell morphology was evaluated and then the culture medium was replaced with $4 \mathrm{ml}$ of fresh medium with puromycin selection factor $(0.5 \mu \mathrm{g} / \mathrm{ml})$ (InvivoGen), and a 2-day selection was performed. Subsequently, the vitality of the cells was evaluated. Moreover, selected PFFs were cultivated with a complete medium without antibiotics until they reached full confluence, in order to perform their molecular analysis.

\section{Genomic Cleavage Assay}

The detection of locus specific cleavage of genomic DNA was performed by a GeneArt Genomic Cleavage Detection Kit as described in the manual. After transfection and selection, PFFs were harvested and washed with phosphate buffered saline (PBS, Sigma-Aldrich). Cells were lysed with $50 \mu \mathrm{l} \mathrm{Cell} \mathrm{Lysis} \mathrm{Buffer}$ and $2 \mu \mathrm{l}$ Protein Degrader at $68^{\circ} \mathrm{C}$ for $15 \mathrm{~min}$; then, the mixture was held at $95{ }^{\circ} \mathrm{C}$ for $10 \mathrm{~min}$. PCR was performed in $50 \mu$ reactions containing $2 \mu$ of cell lysate, $25 \mu \mathrm{l}$ AmpliTaq Gold 360 Master Mix and $10 \mu \mathrm{M}$ forward and reverse primers. For flanking the target site, a forward primer: 5'-TTCAACATGAAAGGGAAGGAG GTA-3' and a reverse primer: 5'-CCTCAATTCACCCAG 
TCTCTTGAT- $3^{\prime}$ were used. The PCR was conducted in the following conditions: $95{ }^{\circ} \mathrm{C}, 10 \mathrm{~min}$ for one cycle; $95^{\circ} \mathrm{C}$, $30 \mathrm{~s} ; 55^{\circ} \mathrm{C}, 30 \mathrm{~s} ; 72{ }^{\circ} \mathrm{C}, 30 \mathrm{~s}$ for a total of 40 cycles; $72{ }^{\circ} \mathrm{C}$, $7 \mathrm{~min}$ for one cycle. The PCR product of $505 \mathrm{bp}(3 \mu \mathrm{l})$ was mixed with $1 \mu \mathrm{l} 10 \times$ Detection Reaction Buffer and $5 \mu \mathrm{l}$ water and then was melted at $95^{\circ} \mathrm{C}$ for $5 \mathrm{~min}$ and cooled at in the following conditions: $95-85^{\circ} \mathrm{C}\left(0,2{ }^{\circ} \mathrm{C} / \mathrm{s}\right), 85-25{ }^{\circ} \mathrm{C}$ $\left(0,1{ }^{\circ} \mathrm{C} / \mathrm{s}\right), 4^{\circ} \mathrm{C}$ for $5 \mathrm{~min}$ in order to form heteroduplexes. Subsequently, the heteroduplex DNA was treated with and without $1 \mu \mathrm{l}$ of the Detection Enzyme at $37^{\circ} \mathrm{C}$ for $1 \mathrm{~h}$ and resolved on a $2 \%$ TBE-agarose gel.

\section{Quantitative Assessment of Genome Editing by Sequence Trace Decomposition}

The CRISPR/Cas9 genome editing efficacy was quantified by indel (insertions and deletions) frequencies. The PCR product obtained under the conditions described above was purified using the StrataPrep PCR Purification Kits (Qiagen) and sequenced using automated genetic analyzers (Applied Biosystems Prism). The sequencing data were analyzed by Tracking of Indels by Decomposition (TIDE) web tool (https ://tide.deskgen.com/), initially described by Brinkman and colleagues [19]. A reference sequence (non-edited chromatograms) was used as a control. Frequencies of indels in cells were determined by the occurrence of significant insertions and deletions in the target site.

\section{Cytotoxicity Test}

To assess the cytotoxicity of the magnetic transfection complexes on PFFs, a Cell Counting Kit-8 (CCK-8, Sigma Aldrich) was used. CCK-8 is a sensitive colorimetric assay allowing the determination of the number of viable cells. CCK- 8 uses water-soluble tetrazolium salt WST- 8 (2-(2-methoxy-4-nitrophenyl)-3-(4-nitrophenyl)-5-(2,4disulfophenyl)-2H-tetrazolium, monosodium salt), which is reduced by dehydrogenases in cells to a formazan dye soluble in the culture medium. The amount of the yellow colored product is directly proportional to the number of living cells. One day prior to transfection, $150 \mu$ l of the PFF cells was seeded at a density of $3 \times 10^{3}$ cells/well in 96-well plate and cultured in a complete medium. On the day of transfection, $50 \mu \mathrm{l}$ of the complexes prepared at a transfection reagent-to-plasmid DNA ratio of $4: 1(\mathrm{v} / \mathrm{w})$ and an ironto-plasmid DNA ratio of 2:1 (w/w) was added to the cells and the plate was incubated on the Mega Magnetic Plate for $20 \mathrm{~min}$. After $24 \mathrm{~h}$ of incubation with the complexes in a total volume of $200 \mu \mathrm{l}, 20 \mu \mathrm{l}$ of the CCK-8 reagent was added to each well; then, the cells were incubated for $1 \mathrm{~h}$ at $37^{\circ} \mathrm{C}$. The spectrophotometric absorbance of each sample was measured by use a microplate reader at $450 \mathrm{~nm}$. The untransfected cells and the cells after lipofection were used as a reference. These experiments were carried out six times, and the results were averaged. The viability of PFFs in each test group was calculated.

\section{Statistical Analysis}

The results were tested for normal distribution (Shapiro test) and homogeneity of variance (Bartlett test). For comparison between mean values, the ANOVA test was used, with Tukey's HSD test for post hoc analysis for data with homogeneity of variance. For data without homogeneity of variance, the Kruskal-Wallis rank sum test and Wilcoxon rank sum test were used. Values of $p<0.05$ were considered as significant. All statistical analyses were performed using $\mathrm{R}$ version 3.4.1.

\section{Results}

To test the potential of magnetofection to improve delivery of CRISPR/Cas9 plasmid into PFFs, we compared this method to classic lipofection using DreamFect Gold transfection reagent. The plasmid for sgRNA and Cas9 expression inducing DNA cleavage at porcine $\mathrm{H} 11$ locus was used for transfection. We tested two plasmid concentrations: $10 \mathrm{pg}$ DNA/cell and $20 \mathrm{pg}$ DNA/cell. After the selection process, PFF cells were harvested and transfection efficiency was evaluated. Targeted double-strand DNA cutting by Cas9 nuclease was tested by a GeneArt Genomic Cleavage Detection Kit. This simple and rapid method uses genomic DNA extracted from cells transfected with CRISPR/Cas9 constructs. Following cleavage, genomic indels are generated by the cellular repair mechanisms. The locus being investigated was amplified by PCR, then denatured and reannealed so that mismatches were created. These mismatches were detected and digested by a Detection Enzyme. The bands obtained were analyzed by gel electrophoresis. The samples treated with Detection Enzyme consisted of the total DNA divided into three bands: two cleaved bands and parental band. As shown in Fig. 2, an advantage was revealed in transfection efficiency (as determined by cleaved bands intensities) arising from the use of magnetofection compared to lipofection in porcine fetal fibroblast cells. Furthermore, heteroduplex bands were only detected in the PCR products from CRISPR-edited cells, but not from wild-type genomes.

A more detailed analysis of CRISPR/Cas9 genome editing efficacy was carried out using the TIDE web tool. The TIDE software precisely determines the spectrum and frequency of indels from the sequence traces. For this purpose, the PCR products of targeted regions from edited and nontransfected cells were sequenced by Sanger's method and sequence trace data were analyzed. As depicted in Fig. 3, the transfection efficiency, measured as indel frequencies, 


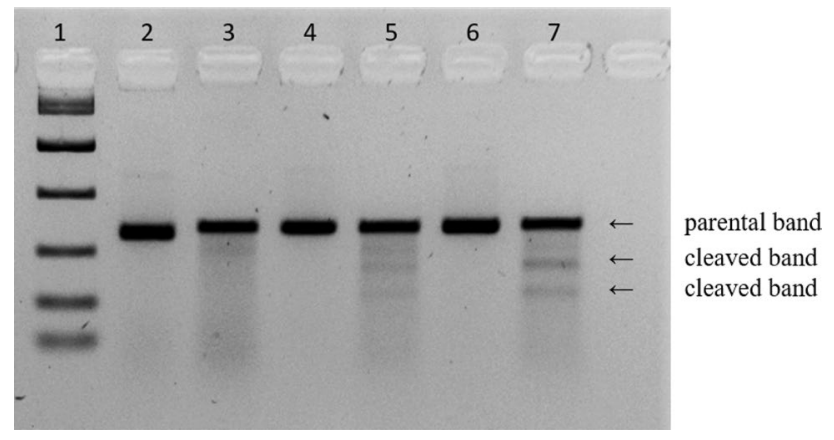

Fig. 2 Gel image of genomic cleavage detection assay using transfected cells. The porcine fetal fibroblast cell lines were transfected with CRISPR/Cas9 plasmid using magnetofection (lanes 6-7) and classic lipofection (lanes 4-5). A negative control sample for gene modification was wild-type genome (lanes 2-3). The samples were PCR amplified using the same set of primers flanking the target site. After re-annealing, samples were treated with (lanes 3, 5, 7) and without a (lanes 2, 4, 6) Detection Enzyme. The separation of DNA fragments was conducted in $2 \%$ agarose gel with size marker (KAPA Express DNA Ladder) (lane 1). The samples digested by a Detection Enzyme consisting of the total DNA divided into three bands: two cleaved bands and parental band

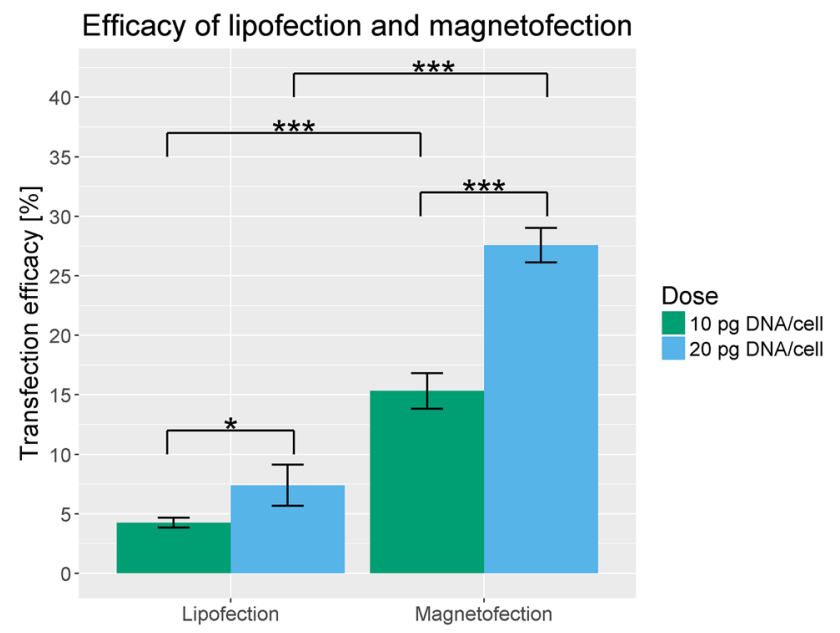

Fig. 3 CRISPR/Cas9 genome editing efficacy quantified by significant indel production frequencies in the target site. The percentage of transfection efficiency in each tested group was marked on Y-axis. More than 3.5-times higher efficacy of genome cleavage occurred in the case of magnetofection (15.3\% for $10 \mathrm{pg}$ DNA/cell; $27.6 \%$ for $20 \mathrm{pg} \mathrm{DNA} /$ cell) compared to lipofection (4.3\% for $10 \mathrm{pg} \mathrm{DNA} / \mathrm{cell}$; $7.4 \%$ for 20 pg DNA/cell). Significance level: $* p<0.05$, $* * p<0.01$, $* * * p<0.001$

was highest when using MNPs $(15.3 \%, 27.6 \%)$ compared to classic lipofection $(4.3 \%, 7.4 \%)$ for both plasmid concentrations, respectively. Moreover, a higher dose of CRISPR/Cas9 construct resulted in increased efficacy of genome cleavage in the target site. Data are presented as mean values from three biological replicates. The differences in the frequency of the desired editing event in transfected cells between magnetofection and lipofection were statistically significant. The TIDE assay identified the predominant types of indels in the DNA of the targeted cells. In this study, we observed that small insertions and deletions appear to be the most common mutations generated by the CRIPSR/Cas9 system. Examples of indels spectrum are shown in Fig. 4.

We did not observe any differences in cell morphology at $24 \mathrm{~h}$ post-transfection between magnetofection and lipofection. Representative microscopy images of magnetofected cells and cells after lipofection are shown in Fig. 5. The cytotoxicity of the MNPs on PFFs was estimated by CCK- 8 colorimetric assay. The survival rate of fibroblasts after transfection was determined. The results of absorbance measurements led to the conclusion that there is no additional cytotoxicity associated with the MNPs compared with the non-magnetic lipoplexes within the tested DNA concentration in the porcine fibroblasts. However, the use of a higher plasmid dose reduced the viability of the PFFs for both transfection methods (for magnetofection from 66.7 to 39.7\%; for lipofection from 65.8 to 39.6\%) (Fig. 6).

\section{Discussion}

An efficient CRISPR/Cas9 system delivery into porcine primary fibroblasts is a prerequisite for successful precise gene modification. In recent years, many non-viral vectors, such as lipids, liposomes and polymeric nanoparticles, have been rapidly developed for this purpose [3]. In our previous work [13], we demonstrated rapid and efficient delivery of a gene construct into PFFs using magnetofection technology. We achieved stable porcine transgenic cell lines expressing human CD59 membrane proteins. The cells were characterized by high proliferative activities and viability, as well as by the correct chromosome number. On this basis, we proposed application of MNPs in combination with a gradient magnetic field for delivery of CRISPR/Cas9 construct into PFFs. To our knowledge, this is the first application of this method to deliver the CRISPR/Cas9 system in order to achieve genome editing. We used PEI-Mag2 iron oxide nanoparticles for assembly into magnetic delivery complexes with CRISPR/Cas9 plasmid and an enhancer. Grześkowiak and colleagues showed that plasmid DNA with PEI-Mag2 nanoparticles and DF-Gold as an enhancer in combination with an applied magnetic field facilitates vector internalization, endosomal escape and protects the nucleic acid against nuclease degradation [20]. In this study, a CRISPR/Cas9 construct expressing the sgRNA and the Cas9 nuclease was prepared to induce site-specific cutting at the porcine H11 locus. A GeneArt Genomic Cleavage Detection Assay was used to obtain an initial estimate of whether targeted doublestrand DNA cutting by Cas9 nuclease was successful or not. 
Fig. 4 Example results of the spectrum of dominant indel types in the cells after magnetofection-a dose of 10 (a)/20 (b) pg DNA/cell and for lipofection-a dose of 10 (c)/20 (d) pg DNA/cell, obtained by means of TIDE software. Bars indicate the percentage of sequences with a given deletion and insertion size. The default range of indel size for the TIDE algorithm is -10 to +10 nucleotides. The percentage sum is $<100$ because nonsignificant peaks are not shown

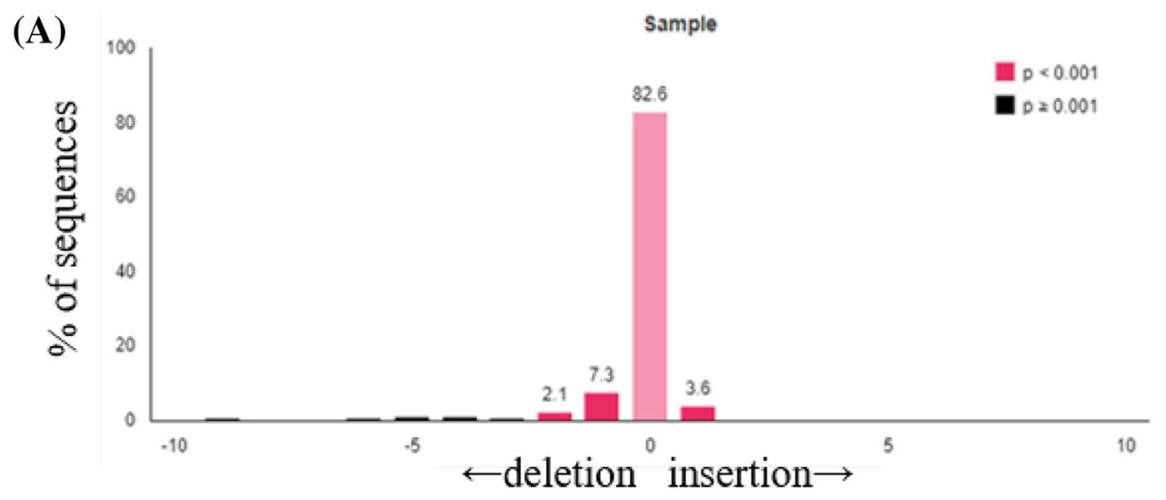

(B)

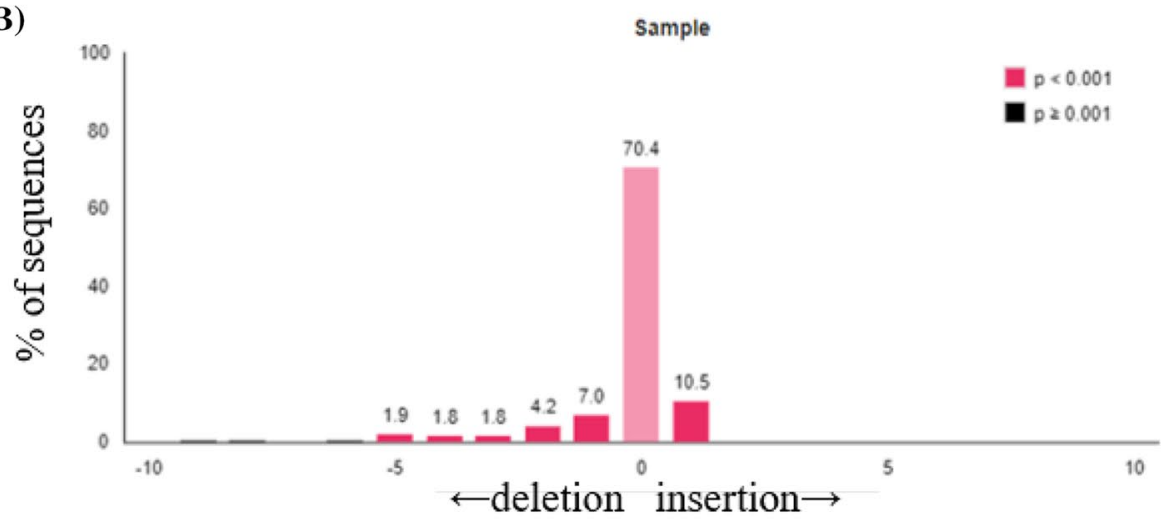

(C)
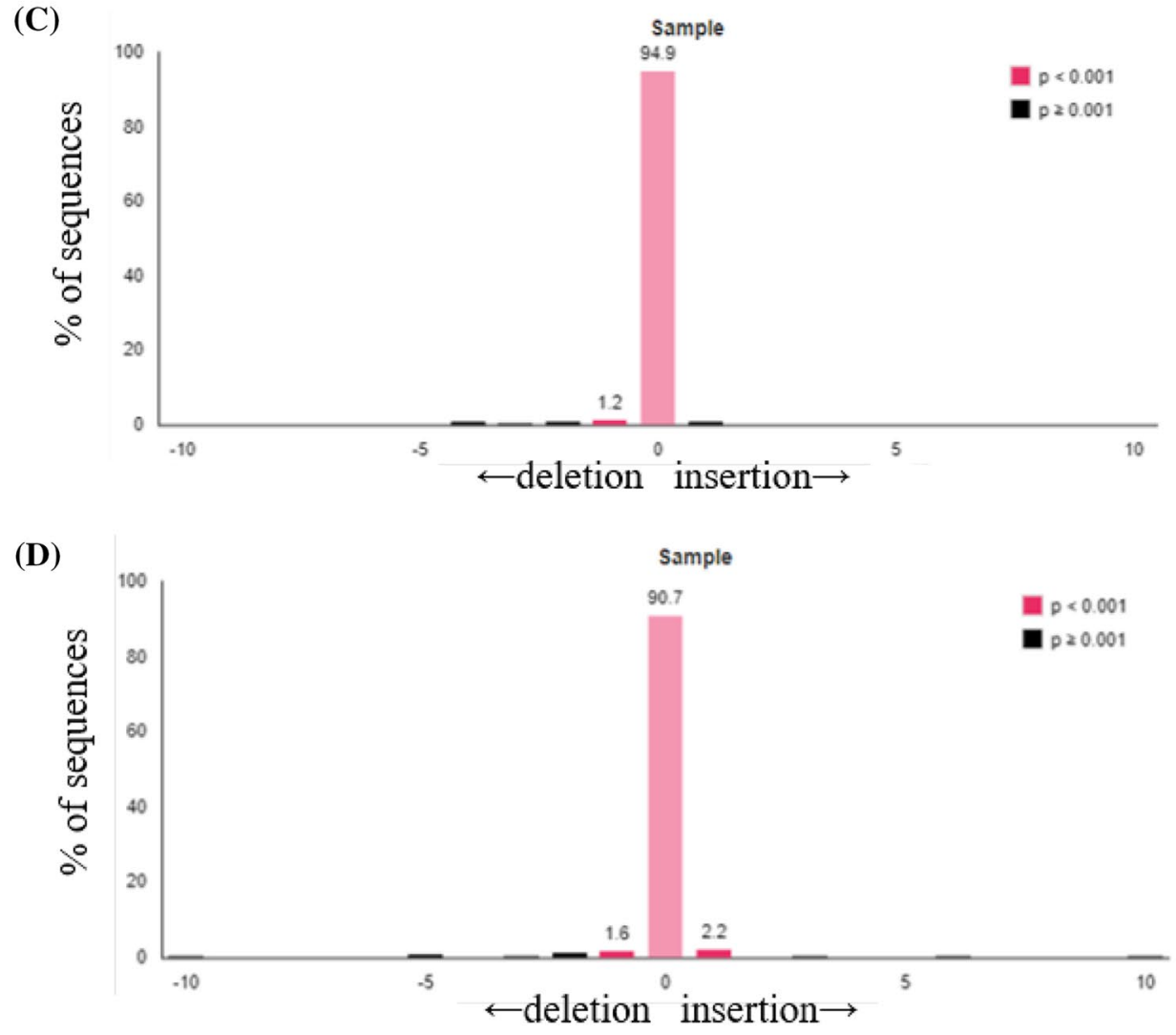

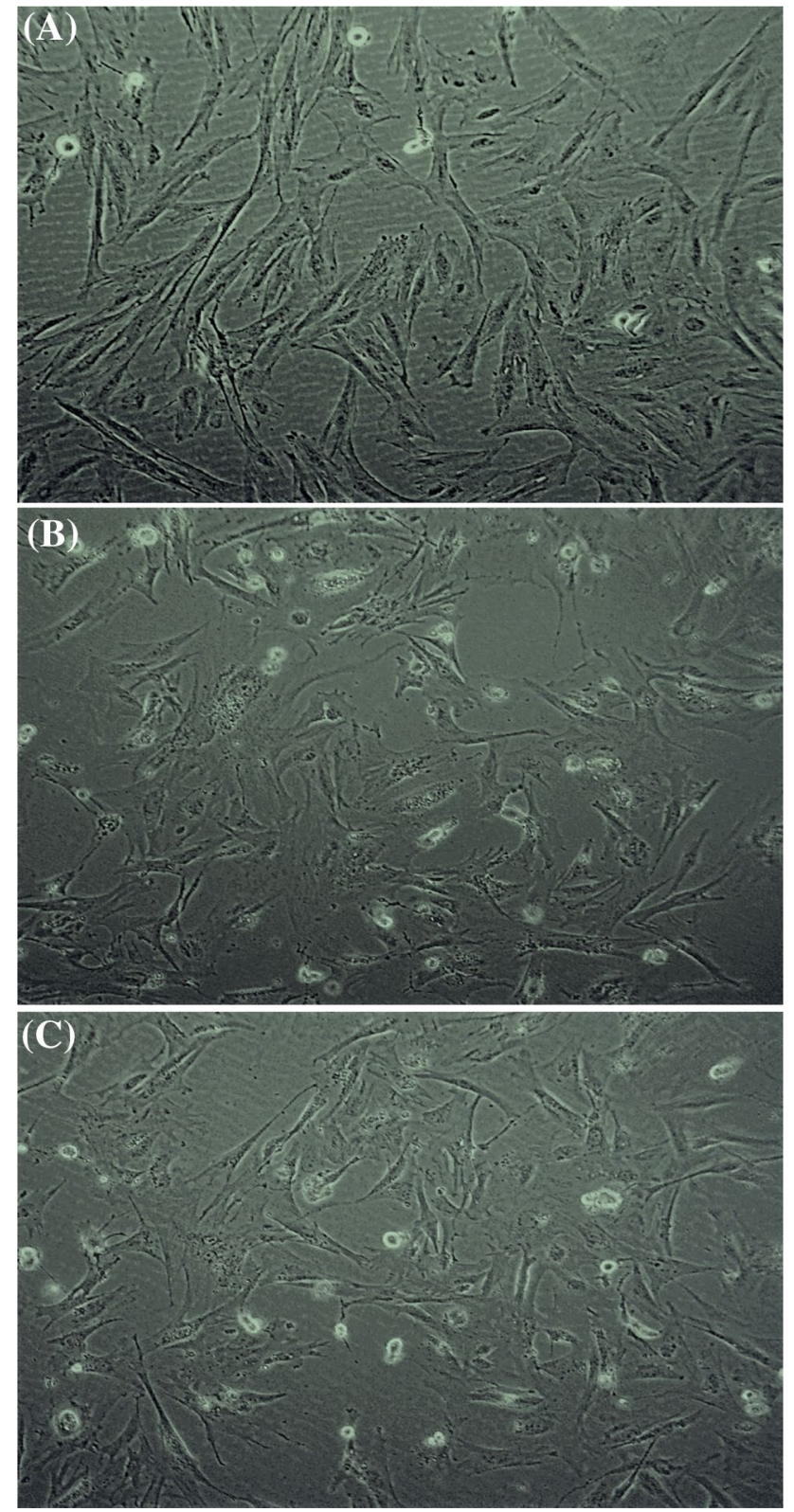

Fig. 5 Microscopy images $(\times 100$ magnification $)$ of non-transfected cells (a), cells $24 \mathrm{~h}$ after lipofection (b), cells $24 \mathrm{~h}$ after magnetofection (c). There were no differences in cell morphology between magnetofected and lipofected fibroblasts. Most of the cells looked healthy and only few floating dead cells were observed

This method confirmed that specific cleavage occurred both after magnetofection and lipofection of CRISPR/Cas9 into PFFs. The levels of editing efficiency at the target site for pools of cells, measured as indel frequencies, were quantified by TIDE web tool. We demonstrated more than 3.5 times higher efficacy of CRISPR/Cas9-mediated genome cleavage occurring in the case of magnetofection compared to the classic lipofection method. The improved transfection efficacy when utilize PEI-Mag2 nanoparticles probably

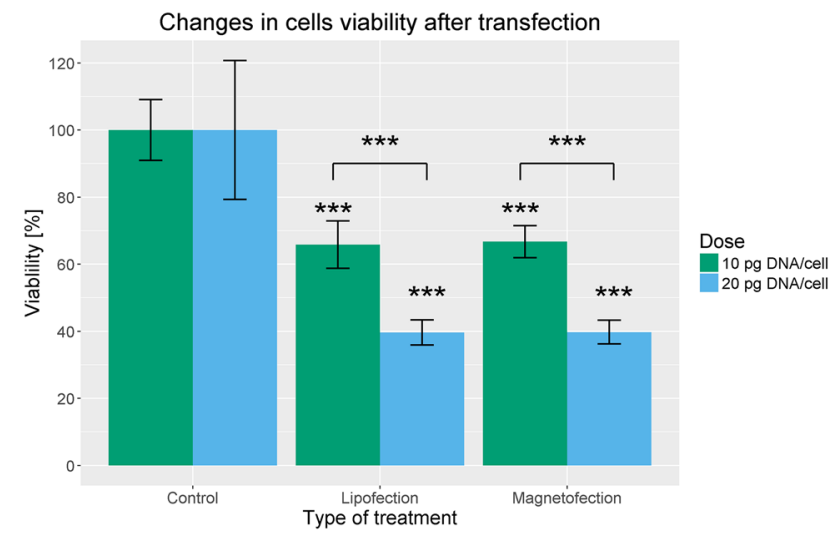

Fig. 6 Survival rate analysis for cells after magnetofection and lipofection. CCK-8 was used to detect the remaining vital cells. The percentage of surviving cells in each group tested was marked on the Y-axis. There were no differences between the level of cell viability that occurred when MNPs complexes $(66.7 \%$ for $10 \mathrm{pg}$ DNA/cell, $39.7 \%$ for $20 \mathrm{pg}$ DNA/cell) were used compared to the non-magnetic lipoplexes (65.8\% for $10 \mathrm{pg} \mathrm{DNA} / \mathrm{cell}, 39.6 \%$ for $20 \mathrm{pg} \mathrm{DNA} / \mathrm{cell}$ ) within the DNA concentration in the porcine fibroblasts that was being tested. Significance level: $* * * p<0.001$

results from their magnetic properties accelerating sedimentation and enhancing concentrations of magnetic transfection complexes on the cell surface in the presence of the magnetic field. Moreover, higher magnetofection efficiency can be caused by reduced non-specific interactions between MNPs and serum components from the cell culture medium, resulting in cellular internalization of the magnetic transfection complexes [18]. The fact that percentages of indel formation in PFF cells were generally not too high $(15.3 \% / 27.6 \%$ for the magnetofection method and $4.3 \% / 7.4 \%$ for lipofection for plasmid doses of 10 and $20 \mathrm{pg} /$ cell, respectively) may result from the sgRNA sequence used in this study, because it is known that the efficiency of CRISPR/Cas9 editing also depends on the choice of sgRNA sites. Nevertheless, the present study clearly indicates that the use of PEI-Mag2 nanoparticles significantly increases the efficiency of ontarget activity compared with non-magnetic lipoplexes. Moreover, a TIDE assay provided an insight in the indel spectrum that occurred in the targeted pool of cells. Overall, small insertions and deletions appear to be most common CRISPR/Cas9-induced mutations. These observations coincide with other studies [19, 21]. The biggest differences among the insertions and deletions events occurred in cell populations edited with a higher dose of CRISPR/Cas9 plasmid in the case of magnetofection (the highest editing efficiency). The most frequently detected type of mutation was a $1 \mathrm{bp}$ insertion, followed by deletions of increasing size. The transfected porcine primary fibroblast cell lines were also characterized by proliferative activities and survival rates. No morphological differences were observed between magnetofected and lipofected cells and a similar level of cell 
viability occurred when MNPs and non-magnetic lipoplexes were used. As expected, the use of a higher plasmid dose increased CRISPR/Cas9 editing efficiency at the expense of decreased cell viability, regardless of the transfection method used.

\section{Conclusion}

The CRISPR/Cas9 system has become one of the most popular genome editing methods used in molecular biology. The efficacy of CRISPR/Cas9 genome editing depends largely on delivery of the CRISPR/Cas9 components into the target cells. In this study, we suggest magnetofection to be an alternative technique for viral and non-viral CRISPR/Cas9 delivery system into porcine fibroblasts. The use of PEI-Mag2 iron oxide nanoparticles in combination with an inhomogeneous magnetic field proved to be an effective, rapid and non-toxic strategy to improve CRISPR/Cas9-mediated on-target activity. Further studies are needed to determine whether the utilization of MNPs adapted for delivery of a CRISPR/Cas9 genome-editing system into porcine fibroblast cell lines can also be used in in vivo conditions.

Acknowledgements This work was supported by The National Centre for Research and Development (Grant No. INNOMED/I/17/ NCBR/2014) in the framework of the "INNOMED" programme titled "Development of an innovative technology using transgenic porcine tissues for biomedical purposes". Acronym: "MEDPIG".

Open Access This article is distributed under the terms of the Creative Commons Attribution 4.0 International License (http://creativeco mmons.org/licenses/by/4.0/), which permits unrestricted use, distribution, and reproduction in any medium, provided you give appropriate credit to the original author(s) and the source, provide a link to the Creative Commons license, and indicate if changes were made.

\section{References}

1. Jinek, M., Chylinski, K., Fonfara, I., Hauer, M., Doudna, J. A., \&Charpentier, E. (2012). A programmable dual-RNAguided DNA endonuclease in adaptive bacterial immunity. Science,337(6096), 816-821.

2. Hryhorowicz, M., Lipiński, D., Zeyland, J., \&Słomski, R. (2017). CRISPR/Cas 9 immune system as a tool for genome engineering. Archivum Immunologiae et Therapiae Experimentalis,65, 233-240.

3. Li, L., Hu, S., \&Chen, X. (2018). Non-viral delivery systems for CRISPR/Cas9-based genome editing: Challenges and opportunities. Biomaterials, 171, 207-218.

4. Glass, Z., Lee, M., Li, Y., \&Xu, Q. (2018). Engineering the delivery system for CRISPR-based genome editing. Trends in Biotechnology,36, 173-185.

5. Mellott, A. J., Forrest, M. L., \&Detamore, M. S. (2013). Physical non-viral gene delivery methods for tissue engineering. Annals of Biomedical Engineering,41, 446-468.
6. Wang, M., Glass, Z. A., \&Xu, Q. (2017). Non-viral delivery of genome-editing nucleases for gene therapy. Gene Therapy,24, 144-150.

7. Alin, Q. U., Chung, J. Y., \&Kim, Y. H. (2015). Current and future delivery systems for engineered nucleases: ZFN, TALEN and RGEN. Journal of Controlled Release, 205, 120-127.

8. Ramakrishna, S., Kwaku Dad, A. B., Beloor, J., Gopalappa, R., Lee, S. K., \&Kim, H. (2014). Gene disruption by cell-penetrating peptide-mediated delivery of Cas9 protein and guide RNA. Genome Research,24(6), 1020-1027.

9. Mout, R., Ray, M., Yesilbag Tonga, G., Lee, Y. W., Tay, T., Sasaki, K., \&Rotello, V. M. (2017). Direct cytosolic delivery of CRISPR/Cas9-ribonucleoprotein for efficient gene editing. ACS Nano, 11(3), 2452-2458.

10. Yue, H., Zhou, X., Cheng, M., \&Xing, D. (2018). Graphene oxide-mediated Cas9/sgRNA delivery for efficient genome editing. Nanoscale, 10(3), 1063-1071.

11. Plank, C., Schillinger, U., Scherer, F., Bergemann, C., Remy, J. S., Krotz, F., et al. (2003). The magnetofection method: using magnetic force to enhance gene delivery. Biological Chemistry, 384,737-747.

12. Plank, C., Zelphati, O., \&Mykhaylyk, O. (2011). Magnetically enhanced nucleic acid delivery. Ten years of magnetofectionprogress and prospects. Advanced Drug Delivery Reviews, 63,1300-1331.

13. Grześkowiak, B. F., Hryhorowicz, M., Tuśnio, K., Grzeszkowiak, M., Załęski, K., Lipiński, D., et al. (2016). Generation of transgenic porcine fibroblast cell lines using nanomagnetic gene delivery vectors. Molecular Biotechnology, 58(5), 351-361.

14. Perleberg, C., Kind, A., \&Schnieke, A. (2018). Genetically engineered pigs as models for human disease. Disease Models \& Mechanisms. https://doi.org/10.1242/dmm.030783

15. Hryhorowicz, M., Zeyland, J., Słomski, R., \&Lipiński, D. (2017). Genetically Modified Pigs as Organ Donors for Xenotransplantation. Molecular Biotechnology, 59(9-10), 435-444.

16. Ruan, J., Li, H., Xu, K., Wu, T., Wei, J., Zhou, R., et al. (2015). Highly efficient CRISPR/Cas9-mediated transgene knockin at the H11 locus in pigs. Scientific Reports, 5,14253.

17. Ran, F. A., Hsu, P. D., Wright, J., Agarwala, V., Scott, D. A., \&Zhang, F. (2013). Genome engineering using the CRISPR-Cas9 system. Nature Protocols, 8,2281-2308.

18. Mykhaylyk, O., Antequera, Y. S., Vlaskou, D., \&Plank, C. (2007). Generation of magnetic nonviral gene transfer agents and magnetofection in vitro. Nature Protocols, 2,2391-2411.

19. Brinkman, E. K., Chen, T., Amendola, M., \&vanSteensel, B. (2014). Easy quantitative assessment of genome editing by sequence trace decomposition. Nucleic Acids Research, 42(22), e168.

20. Grzeskowiak, B. F., Sanchez-Antequera, Y., Hammerschmid, E., Doblinger, M., Eberbeck, D., Wozniak, A., et al. (2015). Nanomagnetic activation as a way to control the efficacy of nucleic acid delivery. Pharmaceutical Research, 32,103-121.

21. Canver, M. C., Bauer, D. E., Dass, A., Yien, Y. Y., Chung, J., Masuda, T., et al. (2014). Characterization of genomic deletion efficiency mediated by CRISPR/Cas9 in mammalian cells. The Journal of Biological Chemistry, 289,21312-21324.

Publisher's Note Springer Nature remains neutral with regard to jurisdictional claims in published maps and institutional affiliations. 